\title{
Setting confidence limits to genetic parameters estimated by restricted maximum likelihood analysis of North Carolina design II experiments
}

\author{
TREVOR HOHLS \\ Department of Genetics, University of Natal, Private Bag X01, Scottsville, 3209, South Africa
}

\begin{abstract}
The properties of the sampling distributions of additive and nonadditive genetic variances and narrow-sense heritabilities were investigated by means of a computer simulation study. Commonly used formulae for the standard errors of these quantities have been tested by means of the coverage probabilities of confidence intervals set using them. Approximate confidence intervals were set assuming a Normal distribution. Formulae that are based on the 'delta' technique, and are shown to produce reliable estimates of the standard errors of additive and nonadditive genetic variances and narrow-sense heritabilities, are provided. Confidence intervals for estimates of the narrow-sense heritability, based on the $F$-distribution, were tested and found to be conservative. A worked example provides an empirical appraisal of the methods of setting confidence limits to genetic parameters estimated by restricted maximum likelihood analysis of North Carolina design II experiments. Suitable statistical software is suggested for obtaining the standard errors of the genetic parameters by the 'delta' technique.
\end{abstract}

Keywords: computer simulation, error rates, genetic variance components, narrow-sense heritability, quantitative genetics, statistical software procedures.

\section{Introduction}

The North Carolina designs I and II (Comstock \& Robinson, 1948) are frequently used in plant breeding experiments to obtain estimates of genetic variance components and heritability. The popularity of these designs is because of their amenability to standard statistical procedures and the ease with which interpretations of variance components can be made in terms of covariances of relatives (Nyquist, 1991).

In the North Carolina design II (Comstock \& Robinson, 1948) or factorial mating design, a.b fullsib families are produced by crossing $\mathbf{a}$ females to $\mathbf{b}$ males. The North Carolina design II produces both half-sib and full-sib families. Half-sib relationships exist for individuals within each female and male parental array, whereas each single-cross is a full-sib.

Narrow-sense heritability, defined as the ratio of additive genetic variance $\left(V_{\mathrm{A}}\right)$ to the phenotypic variance $\left(V_{\mathrm{P}}\right)$ of individuals in the population, provides a means of predicting the response resulting from selection. The narrow-sense heritability $\left(h^{2}\right)$ of plants may be estimated according to several methods, depending on the genotypic structure of the population to which the plants belong, the type of artificial selection used, and the linear model (Hanson, 1963; Knapp et al., 1985; Nyquist, 1991). Heritabilities also differ, depending on whether the reference unit is an individual plant or a plot mean, i.e. heritability on a progeny mean basis (Hanson, 1963).

Appropriate confidence intervals must be given to indicate the accuracy of the estimators of the genetic variance components and $h^{2}$. Standard errors (SEs) and confidence limits are frequently misleading or omitted (Knapp et al., 1985). Hallauer \& Miranda (1988) provide formulae for the variance of additive and nonadditive genetic variance components and $h^{2}$ on an entry mean basis. These formulae are based on Satterthwaite's (1946) approximation for the variance of a variance component. The 'delta' technique (Taylor approximation) has also been used to obtain the standard deviation of a heritability estimate 
(Gordon et al., 1972; Magnussen, 1992). Exact confidence intervals have been developed for $h^{2}$ calculated on a full-sib progeny mean basis from a North Carolina design II experiment (Knapp, 1986). The exact confidence intervals are based on the $F$-distribution because the independent ratios of the observed variance components (used in calculating $h^{2}$ ) and their expectations, are distributed as mutually independent chi-square variables (Knapp et al., 1985; Knapp, 1986). Magnussen (1992) has questioned the accuracy of this method of setting confidence limits, because a covariance exists between the mean squares used in obtaining $h^{2}$ when there are shared environments and unbalanced data, and because the degrees of freedom of the chi-squared distributions are approximations (Satterthwaite, 1946).

Maximum likelihood estimates of variance components are biased downwards because they disregard the degrees of freedom used in estimating treatment effects (Thompson \& Welham, 1993). Patterson \& Thompson (1971) developed the method of restricted maximum likelihood (REML), also known as residual maximum likelihood, to avoid this bias in variance component estimates. REML allows for the analysis of unbalanced data or data with more than one source of variation and is widely used for the analysis of agricultural experiments (Robinson, 1987).

In this article, computer simulations have been used to obtain the sampling distributions of $V_{\mathrm{A}}, V_{\mathrm{D}}$ and $h^{2}$, estimated through REML analysis of a $7 \times 7$ North Carolina design II experiment. The properties of the sampling distributions have been investigated and the empirical SEs of the estimates have been compared with SEs obtained from formulae based on the 'delta' technique, and SEs derived from formulae adapted from those given by Hallauer \& Miranda (1988). Coverage probabilities have been determined for confidence intervals calculated assuming a Normal distribution. The accuracy of confidence intervals of $\hat{h}^{2}$ based upon the $F$-distribution has also been evaluated.

\section{Materials and methods}

The simulation study was based on a $7 \times 7$ North Carolina design II experiment with 10 observations per cross. The model upon which the analysis was based was the following

$Y_{i j k}=\mu+f_{i}+m_{j}+(m f)_{i j}+e_{i j k}$

where: $Y_{i j k}=k$ th phenotypic measurement of the cross between inbreds $i$ and $j$ for trait $Y ; \mu=$ population mean; $f_{i}=$ effect of the $i$ th female, $f_{i}$ are $\mathrm{N}\left(\mu_{\mathrm{f}}\right.$, $\left.\sigma_{\mathrm{f}}^{2}\right) ; m_{j}=$ effect of the $j$ th male, $m_{j}$ are $\mathrm{N}\left(\mu_{\mathrm{m}}, \sigma_{\mathrm{m}}^{2}\right)$; $(m f)_{i j}=$ interaction effect obtained in the cross between lines $i$ and $j,(m f)_{i j}$ are $\mathrm{N}\left(\mu_{\mathrm{mf}}, \sigma_{\mathrm{mf}}^{2}\right)$; and $e_{i j k}=$ within-family variation, $\mathrm{N}\left(0, \sigma_{\mathrm{e}}^{2}\right)$. Under the random effects model, $\mu_{\mathrm{f}}, \mu_{\mathrm{m}}$ and $\mu_{\mathrm{mf}}$ (population means of the female and male main effects and interaction effects, respectively) may be taken as zero.

For full-sibs (FS),

$\operatorname{cov}\left(Y_{i j k}, Y_{i j l}\right)=\sigma_{\mathrm{f}}^{2}+\sigma_{\mathrm{m}}^{2}+\sigma_{\mathrm{mf}}^{2}, k \neq l=1 \ldots r$

and for half-sibs (HS),

$\operatorname{cov}\left(Y_{i j k}, Y_{i m l}\right)=\sigma_{\mathrm{f}}^{2}=\sigma_{\mathrm{m}}^{2}$.

Parameters of particular interest are (assuming completely inbred parents, i.e. an inbreeding coeffi-

Table 1 ANOva showing expected MS for the North Carolina design II experiment used in the simulation study

\begin{tabular}{llll}
\hline Source & d.f. & MS & Expected MS \\
\hline Males and Females & $(a+b-2)$ & $M_{\mathrm{m}+\mathrm{f}}$ & $\sigma_{\mathrm{e}}^{2}+r \sigma_{\mathrm{mf}}^{2}+r[(a+b) / 2] \sigma_{\mathrm{m}+\mathrm{f}}^{2}$ \\
$\quad$ Females & $(a-1)$ & $M_{\mathrm{f}}$ & $\sigma_{\mathrm{e}}^{2}+r \sigma_{\mathrm{mf}}^{2}+r b \sigma_{\mathrm{f}}^{2}$ \\
$\quad$ Males & $(b-1)$ & $M_{\mathrm{m}}$ & $\sigma_{\mathrm{e}}^{2}+r \sigma_{\mathrm{mf}}^{2}+r a \sigma_{\mathrm{m}}^{2}$ \\
Interaction & $(a-1)(b-1)$ & $M_{\mathrm{mf}}$ & $\sigma_{\mathrm{e}}^{2}+r \sigma_{\mathrm{mf}}^{2}$ \\
Within-family variation & $a b(r-1)$ & $M_{\mathrm{e}}$ & $\sigma_{\mathrm{e}}^{2}$ \\
Total & $r a b-1$ & & \\
\hline
\end{tabular}

$a$ and $b$ are the number of female and male parents, respectively, and $r$ is the number of observations per cross; the components of variance are: $\sigma_{m+\mathrm{f}}$ attributable to the pooled males and females terms, $\sigma_{\mathrm{f}}^{2}$ attributable to female parents, $\sigma_{\mathrm{m}}^{2}$ attributable to male parents, $\sigma_{\mathrm{mf}}^{2}$ attributable to interactions, and $\sigma_{\mathrm{e}}^{2}$ attributable to within-family variation. 
cient of $F=1$ ):

$V_{\mathrm{A}}=2 \operatorname{cov}(\mathrm{HS})=2 \sigma_{\mathrm{f}}^{2}=2 \sigma_{\mathrm{m}}^{2}$,

$V_{\mathrm{D}}=\operatorname{cov}(\mathrm{FS})-2 \operatorname{cov}(\mathrm{HS})=\sigma_{\mathrm{mf}}^{2}$

and

$h^{2}=\frac{2 \sigma_{\mathrm{m}+\mathrm{f}}^{2}}{\frac{\sigma_{\mathrm{e}}^{2}}{r}+\sigma_{\mathrm{mf}}^{2}+2 \sigma_{\mathrm{m}+\mathrm{f}}^{2}}$,

where $\sigma_{\mathrm{m}+\mathrm{f}}^{2}$ is the variance component for the pooled variation attributable to females and males. $h^{2}$ may also be interpreted as the narrow-sense heritability on a full-sib progeny mean basis.

Table 1 shows the expected values of the mean squares in the traditional analysis of variance.

The sampling distributions of $\hat{V}_{\mathrm{A}}, \hat{V}_{\mathrm{D}}$ and $\hat{h}^{2}$ were investigated by means of a Monte Carlo simulation. Those parameters influencing such distributions (apart from the experimental design itself) are: $\sigma_{\mathrm{e}}^{2}$, $\sigma_{\mathrm{f}}^{2}, \sigma_{\mathrm{m}}^{2}$ and $\sigma_{\mathrm{mf}}^{2}$. Various combinations of values for these four parameters were used in the simulations, as shown in Table 2. One thousand simulations were carried out for each parameter combination. Once a set of random variables had been generated for a simulation a REML analysis was performed using GENSTAT (1993), in which the females, males and interactions were treated as random effects, to obtain the relevant variance components. The variance component estimates were then used to obtain the genetic parameter estimates, viz. $\hat{V}_{\mathrm{A}}, \hat{V}_{\mathrm{D}}$ and $\hat{h}^{2}$, from eqns (4), (5) and (6).

Because the genetic parameters of interest are merely linear functions, or for $h^{2}$ a ratio of two linear functions, of the variance components $\sigma_{\mathrm{f}}^{2}, \sigma_{\mathrm{m}}^{2}$, $\sigma_{\mathrm{mf}}^{2}$ and $\sigma_{\mathrm{e}}^{2}$ (formulae (4)-(6)), estimates of the SEs of $\hat{V}_{\mathrm{A}}, \hat{V}_{\mathrm{D}}$ and $\hat{h}^{2}$ may be obtained using the 'delta' technique for deriving the variance of a linear function (Snedecor \& Cochran, 1989). These formulae are provided in Appendix 1. The estimates of the variance components and their variance-covariance matrix are obtained from the REML analysis. It is noteworthy that GENSTAT 5.31 (1993) has a procedure called VFUNCTION that calculates functions and SEs of functions of variance components obtained from a REML analysis. The SEs of the functions are computed according to the 'delta' method provided in Appendix 1.

Now, using the formula for estimating sampling variances of variance components (Satterthwaite, 1946):

$V\left(\hat{\sigma}_{\mathrm{c}}^{2}\right)=\frac{2}{k^{2}} \sum_{u} \frac{\mathrm{MS}_{u}^{2}}{f_{u}+2}$ (where $k$ is the coefficient of the variance component being estimated $\left(\hat{\sigma}_{c}^{2}\right)$ and $f_{u}$ are the degrees of freedom of the $u$ th Mean Square), Hallauer \& Miranda (1988) derived formulae for approximate SEs of $\hat{V}_{\mathrm{A}}, \hat{V}_{\mathrm{D}}$ and $\hat{h}^{2}$. The formulae for the $\operatorname{SE}\left(\hat{V}_{\mathrm{A}}\right)$, $\operatorname{SE}\left(\hat{V}_{\mathrm{D}}\right)$ and $\operatorname{SE}\left(\hat{h}^{2}\right)$, modified for inbred parents $(F=1)$ and to enable use of the males and females term (as there is an equal number of male and female parents), are provided in Appendix 2.

Confidence limits to $\hat{V}_{\mathrm{A}}, \hat{V}_{\mathrm{D}}$ and $h^{2}$ were based on the Normal distribution and set using the SEs obtained from formulae (A1.1), (A1.2) and (A1.3), and the SEs computed using formulae (A2.1), (A2.2) and (A2.3). Confidence limits for $\hat{h}^{2}$ were also set according to the method based on the $F$-distribution, proposed by Knapp et al. (1985), and modified by Knapp (1986) for two-factor mating designs. Confidence limits to $\hat{h}^{2}$ were obtained for a $(1-\alpha)$ level of significance, by using the following formula (from Knapp, 1986):

$$
\begin{aligned}
\{1+ & {\left.[(a+b) / 4] \cdot\left[\left(M_{\mathrm{m}+\mathrm{f}} / M_{\mathrm{mf}}\right) \cdot F_{1-\alpha / 2: d f(\mathrm{mf}), d f(\mathrm{~m}+\mathrm{f})}-1\right]^{-1}\right\}^{-1} } \\
\leq & h^{2} \leq\left\{1+[(a+b) / 4] \cdot\left[\left(M_{\mathrm{m}+\mathrm{f}} / M_{\mathrm{mf}}\right) \cdot F_{\alpha / 2: d f(\mathrm{mf}), d f(\mathrm{~m}+\mathrm{f})}\right.\right. \\
& \left.-1]^{-1}\right\}^{-1},
\end{aligned}
$$

where $a$ and $b$ are the numbers of female and male parents, respectively. For example, in this experiment, 95 per cent confidence limits were set using the following values calculated by GENSTAT (1993) through interpolation from $F$-tables: $F_{0.975: 36,12}=0.4294$, and $F_{0.025: 36,12}=2.925$.

For each simulated data set, the values of $\hat{V}_{\mathrm{A}}, \hat{V}_{\mathrm{D}}$ and $\hat{h}^{2}$ were recorded. When the 1000 simulations were complete, the log transformed SEs of the estimates and the coverage probabilities of the confidence limits set using all the methods described above, were recorded. The error rate statistic was calculated as the percentage of $\hat{V}_{\mathrm{A}}, \hat{V}_{\mathrm{D}}$ and $\hat{h}^{2}$ that fell outside the confidence limits set using the tested SEs.

Table 2 Parameter combinations used in the computer simulations of a $7 \times 7$ North Carolina design II experiment

\begin{tabular}{lccc}
\hline$\sigma_{\mathrm{e}}^{2}$ & $\sigma_{\mathrm{f}}^{2}$ & $\sigma_{\mathrm{m}}^{2}$ & $\sigma_{\mathrm{mf}}^{2}$ \\
\hline 0.50 & 0.10 & 0.10 & 0.10 \\
1.00 & 0.50 & 0.50 & 0.50 \\
& 1.00 & 1.00 & 1.00 \\
& 1.50 & 1.50 & 1.50 \\
& 3.00 & 3.00 & 3.00 \\
\hline
\end{tabular}



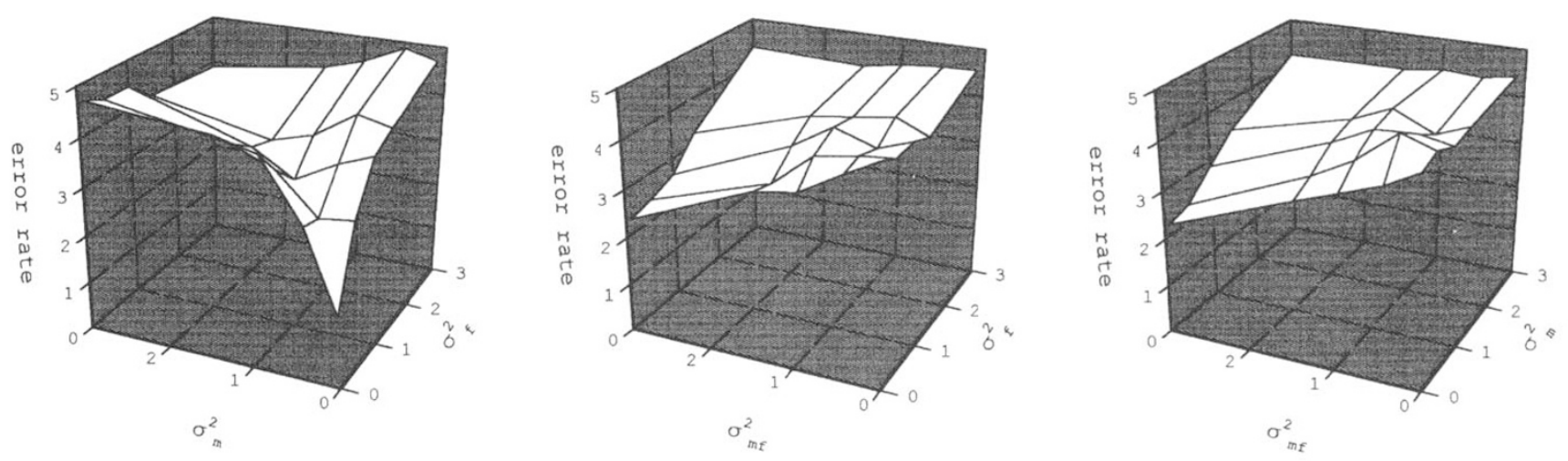

Fig. 1 Relationship between the first-order interactions of the variance components for females $\left(\sigma_{\mathrm{f}}^{2}\right)$, males $\left(\sigma_{\mathrm{m}}^{2}\right)$ and interactions $\left(\sigma_{\mathrm{mf}}^{2}\right)$ for the error rate (per cent) of additive variance estimates $\left(\hat{V}_{\mathrm{A}}\right)$ falling outside confidence limits set using the 'delta' technique.
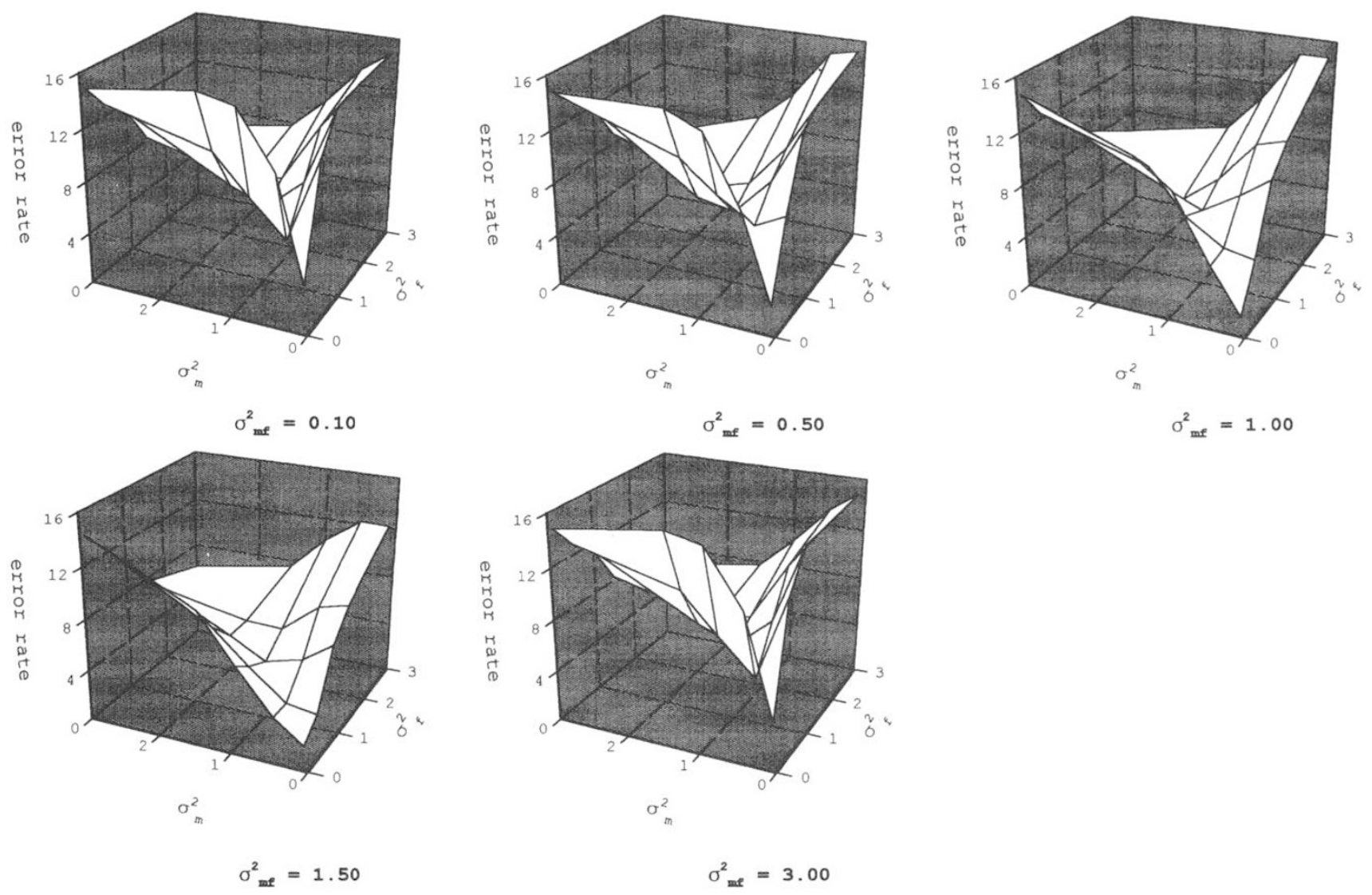

Fig. 2 Relationship between the variance components for females $\left(\sigma_{\mathrm{f}}^{2}\right)$, males $\left(\sigma_{\mathrm{m}}^{2}\right)$ and interactions $\left(\sigma_{\mathrm{mf}}^{2}\right)$ for the error rate (per cent) of additive variance estimates $\left(\hat{V}_{\mathrm{A}}\right)$ falling outside confidence limits set using formulae derived from Hallauer \& Miranda (1988). 
The results of the simulations were summarized factorially for the log transformed SE of the estimates, the $\log$ transformed SE of the estimates calculated using the formulae given in Appendices 1 and 2, and the error rate (per cent) using the various methods of setting confidence limits to the estimates.

A worked example has been included to provide empirical evidence of the differences in the SEs of $\hat{V}_{\mathrm{A}}, \hat{V}_{\mathrm{D}}$ and $\hat{h}^{2}$, depending on the formula used. The example is based on data obtained from a $7 \times 7$ North Carolina design II experiment of maize conducted at Ukulinga Research Farm, Natal, South Africa.

\section{Results}

$\hat{\mathrm{V}}_{A}$

Fewer than 5 per cent of the $\hat{V}_{\mathrm{A}}$ fell outside confidence limits set using SEs computed from formula (A1.1) based on the 'delta' technique (Fig. 1). All three first-order interactions of $\sigma_{\mathrm{f}}^{2}, \sigma_{\mathrm{m}}^{2}$ and $\sigma_{\mathrm{mf}}^{2}$ contributed significantly $(P<0.01)$ to the overall variation in the error rate. Specifically, the interaction between $\sigma_{\mathrm{f}}^{2}$ and $\sigma_{\mathrm{m}}^{2}$ accounted for 32 per cent of the variation in error rate. The highest SEs, and hence widest confidence intervals, were produced when $\sigma_{\mathrm{f}}^{2}$ and $\sigma_{\mathrm{m}}^{2}$ were at their lowest parameter setting of 0.1 (Fig. 1).

An average of 8.3 per cent of $\hat{V}_{\mathrm{A}}$ fell outside confidence limits set using SEs obtained from formula (A2.1) based on a formula given by Hallauer \& Miranda (1988). A significant $(P<0.01)$ portion of the overall variation in the error rate could be accounted for by the second-order interaction between $\sigma_{\mathrm{f}}^{2}, \sigma_{\mathrm{m}}^{2}$ and $\sigma_{\mathrm{mf}}^{2}$, i.e. $\sigma_{\mathrm{f}}^{2} \cdot \sigma_{\mathrm{m}}^{2} \cdot \sigma_{\mathrm{mf}}^{2}$. The error rates of $\hat{V}_{\mathrm{A}}$ falling outside the confidence limits based on Hallauer \& Miranda's (1988) SEs were unacceptably high (Fig. 2). The lowest error rates occurred when $\sigma_{\mathrm{f}}^{2}=\sigma_{\mathrm{m}}^{2}=0.10$ (Fig. 2). The highest error rates were recorded when either $\sigma_{\mathrm{m}}^{2}$ or $\sigma_{\mathrm{f}}^{2}$ was at its highest parameter setting i.e. 3.0 (Fig. 2).

$\hat{\mathrm{V}}_{D}$

Although fewer than 5 per cent of the $\hat{V}_{\mathrm{D}}$ fell outside confidence limits set using SEs computed from formula (A1.2) (using the 'delta' technique), an error rate of up to 6.1 per cent was recorded when SEs were calculated using formula (A2.2) (based on formulae given by Hallauer \& Miranda (1988)). The only parameters that contributed significantly $(P<0.01)$ to the variation in error rate of $\hat{V}_{\mathrm{D}}$ falling outside confidence limits set using SEs obtained from formulae (A1.2) and (A2.2), were the main effects and interaction of $\sigma_{\mathrm{mf}}^{2}$ and $\sigma_{\mathrm{e}}^{2}$. The lowest error rates occurred when $\sigma_{\mathrm{mf}}^{2}=0.1$ and $\sigma_{\mathrm{e}}^{2}=1.0($ Table 3$)$.

\section{$\hat{\mathrm{h}}^{2}$}

Formula (A1.3), which is based on the 'delta' technique, produced SEs that resulted in most of the $\hat{h}^{2}$ falling inside the relevant confidence limits. A significant $(P<0.01)$ portion of the overall variation in the error rate could be accounted for by the secondorder interaction between $\sigma_{\mathrm{f}}^{2}, \sigma_{\mathrm{m}}^{2}$ and $\sigma_{\mathrm{mf}}^{2}$, i.e. $\sigma_{\mathrm{f}}^{2} \cdot \sigma_{\mathrm{m}}^{2} \cdot \sigma_{\mathrm{mf}}^{2}$. Although the error rate went up to more than 7 per cent when either $\sigma_{\mathrm{f}}^{2}$ or $\sigma_{\mathrm{m}}^{2}$ was at the highest parameter setting, i.e. 3.0 (Fig. 3), the average error rate (per cent) of $\hat{h}^{2}$ falling outside confidence limits set using formula (A1.3) was 4.85.

None of the $\hat{h}^{2}$ fell outside the confidence limits set using formula (A2.3). The realized coverage of the confidence limits was wider than expected

Table 3 First-order interaction of female $\times$ male interaction and error variance components $\left(\sigma_{\mathrm{mf}}^{2}, \sigma_{\mathrm{e}}^{2}\right)$ for the errcr rate of $\hat{V}_{\mathrm{D}}$ falling outside the confidence limits set using SEs calculated from the formulae given in Appendices 1 and 2

\begin{tabular}{ll}
\multicolumn{3}{c}{$\sigma_{\mathrm{e}}^{2}$} & \\
\hline 0.5 & 1.0
\end{tabular}

(a) Error rate 1

$\begin{array}{llll}\sigma_{\mathrm{mf}}^{2} & 0.1 & 3.240 & 1.816 \\ & 0.5 & 4.624 & 4.536 \\ & 1.0 & 4.700 & 4.620 \\ & 1.5 & 4.676 & 4.468 \\ & 3.0 & 4.948 & 4.720\end{array}$

(b) Error rate 2

\begin{tabular}{llll}
$\sigma_{\mathrm{mf}}^{2}$ & 0.1 & 3.488 & 2.020 \\
& 0.5 & 5.144 & 5.160 \\
& 1.0 & 5.320 & 5.232 \\
& 1.5 & 5.244 & 5.136 \\
& 3.0 & 5.536 & 5.324 \\
\hline
\end{tabular}

Error rate $1=$ error rate of $\hat{V}_{\mathrm{D}}$ falling outside confidence limits set using SEs calculated using formula A1.2 (based on the 'delta' technique).

Error rate $2=$ error rate of $\hat{V}_{\mathrm{D}}$ falling outside confidence limits set using SEs calculated using formula A2.2 (based on the formula given by Hallauer \& Miranda (1988)).

SED $($ Error rate 1$)=0.1775$; SED $($ Error rate 2) $=0.1927$. 

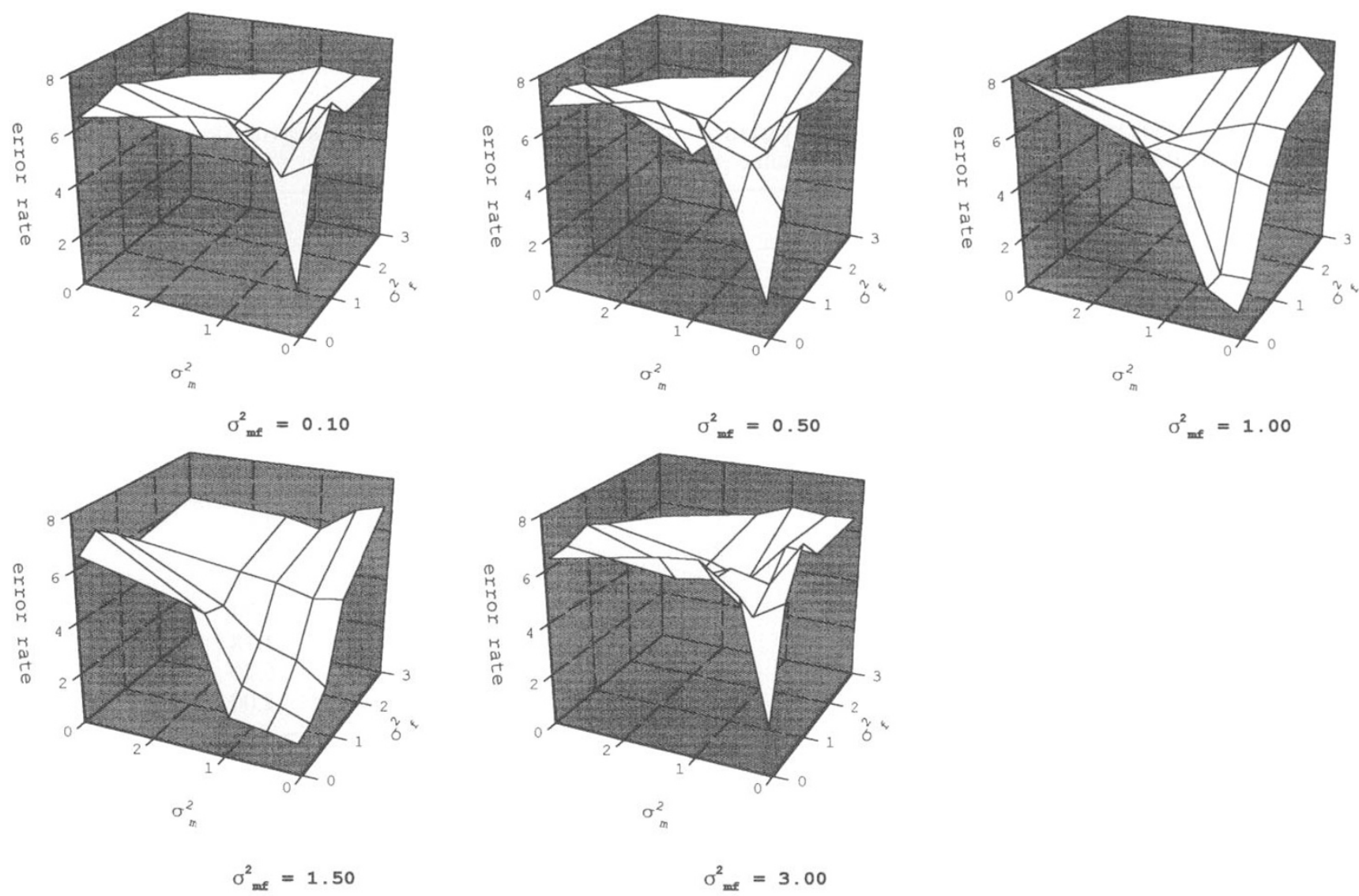

Fig. 3 Relationship between the variance components for females $\left(\sigma_{\mathrm{f}}^{2}\right)$, males $\left(\sigma_{\mathrm{m}}^{2}\right)$ and interactions $\left(\sigma_{\mathrm{mf}}^{2}\right)$ in terms of the error rate (per cent) of heritability estimates $\left(\hat{h}^{2}\right)$ falling outside confidence limits set using the 'delta' technique.

because the SEs produced by formula (A2.3), based on the formula given by Hallauer \& Miranda (1988), were too high. The SEs obtained by formula (A2.3) were much higher than either the empirical SEs or the SEs generated from formula (A1.3) (Table 4). It is noteworthy that the SEs produced by formula (A1.3), based on the 'delta' technique, were almost identical to the empirical SEs (Table 4). Estimates of the $\mathrm{SE}\left(\hat{h}^{2}\right)$ produced by formula (A1.3) were highly correlated with the empirical SEs (0.99, $P<0.001$ ).

The error rates derived by setting confidence limits using formula (8), and based on the $F$-distribution, were unacceptably high (Fig. 4). An average of 8.57 per cent of the $h^{2}$ fell outside the confidence limits set using formula (8), and the error rate went up to 17.3 per cent when $\sigma_{\mathrm{f}}^{2}=0.10$, $\sigma_{\mathrm{m}}^{2}=1.5$, and $\sigma_{\mathrm{mf}}^{2}=0.10$ (Fig. 4). The second-order interaction between $\sigma_{\mathrm{f}}^{2}, \sigma_{\mathrm{m}}^{2}$ and $\sigma_{\mathrm{mf}}^{2}$, i.e. $\sigma_{\mathrm{f}}^{2} \cdot \sigma_{\mathrm{m}}^{2} \cdot \sigma_{\mathrm{mf}}^{2}$, accounted for a highly significant $(P<0.01)$ portion of the overall variation in the error rate. The error rate of $\hat{h}^{2}$ falling outside confidence limits based on the F-distribution was generally higher when either $\sigma_{\mathrm{f}}^{2}$ or $\sigma_{\mathrm{m}}^{2}$ approached their highest parameter setting of 3.0, and lowest when $\sigma_{\mathrm{f}}^{2}=\sigma_{\mathrm{m}}^{2}=0.1$ (Fig. 4).

\section{Worked example}

The $\operatorname{SE}\left(\hat{V}_{\mathrm{A}}\right)$ produced by formula (A1.1) and derived from the 'delta' technique was 8 per cent higher than the SE produced by formula (A2.1) (derived from Hallauer \& Miranda (1988)) (Table 5 ). There was closer agreement between the SEs of $\hat{V}_{\mathrm{D}}$, produced by the 'delta' technique (formula (A1.2) ) and formula (A2.2), which is based on the formula of Hallauer \& Miranda (1988). The SE $\left(\hat{h}^{2}\right)$ obtained by the formula derived from Hallauer \& Miranda (1988) was more than nine times higher 
Table 4 Second-order interaction table of the three variance components $\left(\sigma_{\mathrm{f}}^{2} \cdot \sigma_{\mathrm{m}}^{2} \cdot \sigma_{\mathrm{mf}}^{2}\right)$ for the log transformed SE of $\hat{V}_{\mathrm{A}}$ calculated from 1000 simulation runs and using formulae A1.3 (in bold) and A2.3 (in italics)

\begin{tabular}{|c|c|c|c|c|c|c|}
\hline \multirow[b]{2}{*}{$\sigma_{\mathrm{f}}^{2}$} & \multirow[b]{2}{*}{$\sigma_{\mathrm{m}}^{2}$} & \multicolumn{5}{|c|}{$\sigma_{m f}^{2}$} \\
\hline & & 0.10 & 0.50 & 1.00 & 1.50 & 3.00 \\
\hline \multirow[t]{15}{*}{0.10} & \multirow[t]{3}{*}{0.10} & -2.04 & -2.31 & -2.44 & -2.46 & -2.51 \\
\hline & & -1.83 & -1.89 & -1.91 & -1.92 & -1.92 \\
\hline & & 0.86 & 1.07 & 1.10 & 1.11 & 1.11 \\
\hline & \multirow{3}{*}{0.50} & -2.16 & -1.78 & -1.98 & -2.15 & -2.39 \\
\hline & & -2.28 & -1.78 & -1.80 & -1.86 & -1.90 \\
\hline & & -0.20 & 0.57 & 0.94 & 1.03 & 1.09 \\
\hline & \multirow[t]{3}{*}{1.00} & -2.81 & -1.93 & -1.76 & -1.82 & -2.14 \\
\hline & & -3.11 & -2.10 & -1.78 & -1.77 & -1.86 \\
\hline & & -0.57 & -0.12 & 0.50 & 0.80 & 1.03 \\
\hline & \multirow[t]{3}{*}{1.50} & -3.53 & -2.29 & -1.82 & -1.74 & -1.95 \\
\hline & & -3.79 & -2.53 & -1.94 & -1.78 & -1.80 \\
\hline & & -0.63 & -0.47 & 0.10 & 0.50 & 0.93 \\
\hline & \multirow[t]{3}{*}{3.00} & -4.44 & -3.35 & -2.43 & -2.00 & -1.75 \\
\hline & & -5.05 & -3.59 & -2.63 & -2.18 & -1.78 \\
\hline & & -0.63 & -0.63 & -0.51 & -0.22 & 0.50 \\
\hline \multirow[t]{15}{*}{0.50} & \multirow[t]{3}{*}{0.10} & -2.19 & -1.79 & -1.96 & -2.14 & -2.39 \\
\hline & & -2.28 & -1.78 & -1.81 & -1.86 & -1.90 \\
\hline & & -0.20 & 0.56 & 0.93 & 1.03 & 1.09 \\
\hline & \multirow[t]{3}{*}{0.50} & -2.87 & -1.95 & -1.88 & -2.01 & -2.28 \\
\hline & & -2.90 & -1.99 & -1.81 & -1.84 & -1.89 \\
\hline & & -0.43 & 0.26 & 0.79 & 0.97 & 1.07 \\
\hline & \multirow[t]{3}{*}{1.00} & -3.43 & -2.27 & -1.83 & -1.85 & -2.13 \\
\hline & & -3.53 & -2.34 & -1.87 & -1.79 & -1.85 \\
\hline & & -0.60 & -0.24 & 0.43 & 0.76 & 1.02 \\
\hline & \multirow[t]{3}{*}{1.50} & -3.84 & -2.59 & -1.96 & -1.80 & -1.94 \\
\hline & & -4.04 & -2.74 & -2.03 & -1.82 & -1.81 \\
\hline & & -0.63 & -0.50 & 0.04 & 0.46 & 0.92 \\
\hline & \multirow[t]{3}{*}{3.00} & -4.87 & -3.38 & -2.47 & -2.03 & -1.76 \\
\hline & & -5.18 & -3.66 & -2.70 & -2.20 & -1.79 \\
\hline & & -0.63 & -0.63 & -0.52 & -0.22 & 0.49 \\
\hline \multirow[t]{15}{*}{1.00} & \multirow[t]{3}{*}{0.10} & -2.83 & -1.95 & -1.76 & -1.82 & -2.19 \\
\hline & & -3.11 & -2.10 & -1.78 & -1.77 & -1.86 \\
\hline & & -0.57 & -0.12 & 0.51 & 0.80 & 1.03 \\
\hline & \multirow[t]{3}{*}{0.50} & -3.43 & -2.22 & -1.84 & -1.85 & -2.14 \\
\hline & & -3.54 & -2.33 & -1.87 & -1.79 & -1.85 \\
\hline & & -0.60 & -0.23 & 0.42 & 0.74 & 1.02 \\
\hline & \multirow[t]{3}{*}{1.00} & -4.00 & -2.66 & -2.03 & -1.91 & -2.03 \\
\hline & & -4.05 & -2.71 & -2.04 & -1.85 & -1.83 \\
\hline & & -0.62 & -0.42 & 0.18 & 0.57 & 0.97 \\
\hline & \multirow[t]{3}{*}{1.50} & -4.31 & -2.97 & -2.18 & -1.95 & -1.93 \\
\hline & & -4.46 & $-\mathbf{3 . 0 8}$ & -2.23 & -1.93 & -1.81 \\
\hline & & -0.63 & -0.55 & -0.09 & 0.34 & 0.87 \\
\hline & \multirow[t]{3}{*}{3.00} & -5.09 & -3.64 & -2.69 & -2.18 & -1.78 \\
\hline & & $-\mathbf{5 . 3 7}$ & -3.87 & -2.84 & -2.31 & -1.83 \\
\hline & & -0.63 & -0.63 & -0.53 & -0.26 & 0.45 \\
\hline
\end{tabular}


Table 4 Continued

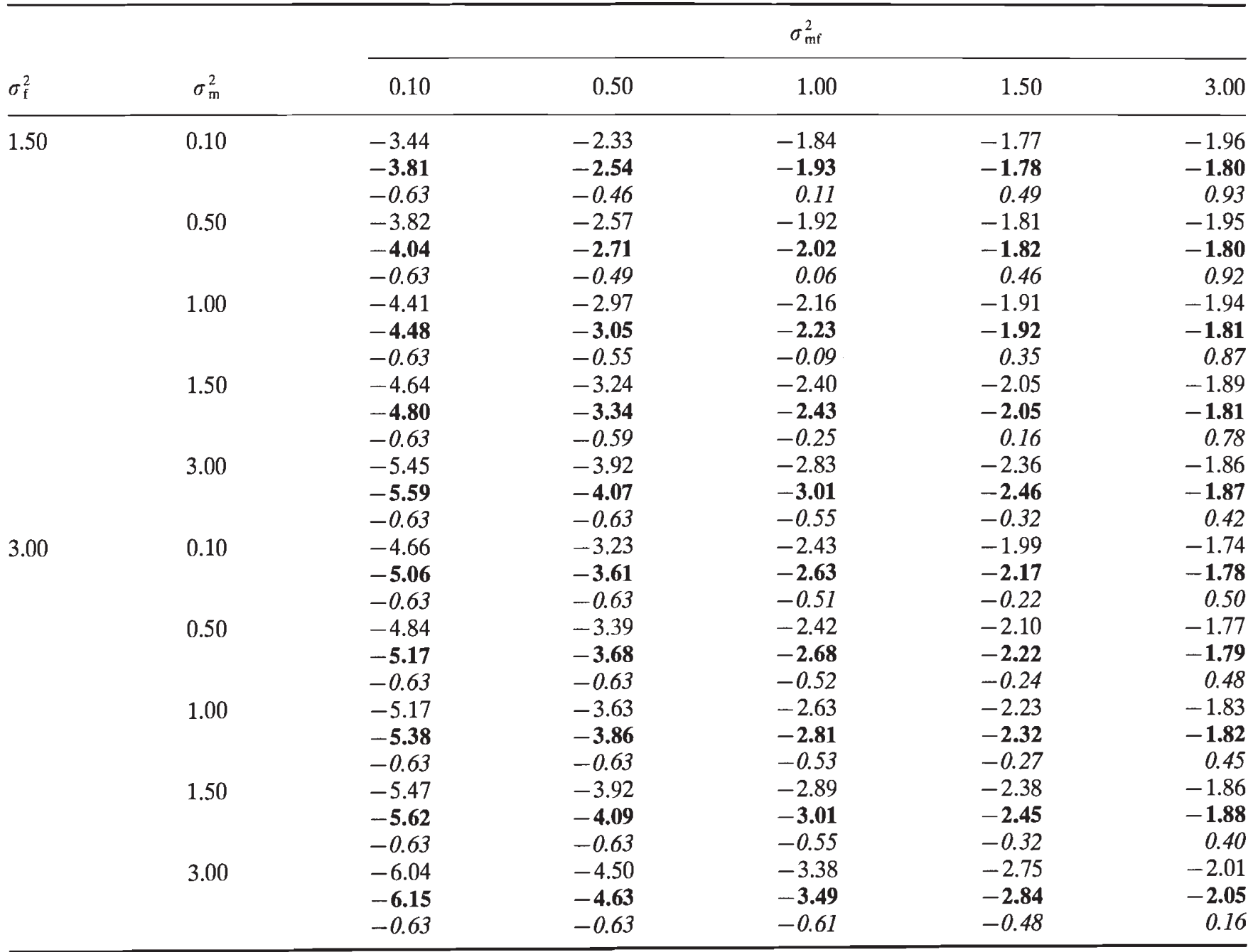

$\operatorname{SED}($ empirical SE) $=0.0856$, SED $($ formula A1.3) $=\mathbf{0 . 0 5 8 6}, S E D($ formula $A 2.3)=0.0422$.

than the SE obtained using the 'delta' technique. The confidence interval constructed using the SE derived from the 'delta' technique overlapped that based on the $F$-distribution (Table 5). The upper confidence limit derived from the $F$-distribution was, however, lower than that generated from the SE computed using the 'delta' technique.

\section{Discussion}

The 'delta' technique provides reliable estimates of the SEs of $\hat{V}_{\mathrm{A}}, \hat{V}_{\mathrm{D}}$ and $\hat{h}^{2}$. The SEs of $\hat{V}_{\mathrm{A}}, \hat{V}_{\mathrm{D}}$ and $\hat{h}^{2}$ obtained using formulae (A1.1), (A1.2) and (A1.3), respectively, were slightly too large, resulting in fewer than 5 per cent of the estimates falling outside the confidence limits (Fig. 1, Table 3, Fig. 3). The realized coverage of the resultant confidence limits was therefore wider than the stated coverage of 95 per cent. These slight overestimates in the SEs of $\hat{V}_{\mathrm{A}}, \hat{V}_{\mathrm{D}}$ and $\hat{h}^{2}$ resulted in 1.15 per cent fewer $\hat{V}_{\mathrm{A}}$, 0.77 per cent fewer $\hat{V}_{\mathrm{D}}$ and 0.15 per cent fewer $\hat{h}^{2}$ than expected falling outside the confidence limits. The results of this study agree with those of Magnussen (1992) who found, using a simulation study of a single-factor sib-analysis trial, that the 'delta' technique provided the best estimates of the standard deviation of the sample heritability. Despite the slight bias in the estimates of the SEs of $\hat{V}_{\mathrm{A}}, \hat{V}_{\mathrm{D}}$ and $\hat{h}^{2}$ obtained using the 'delta' technique, 

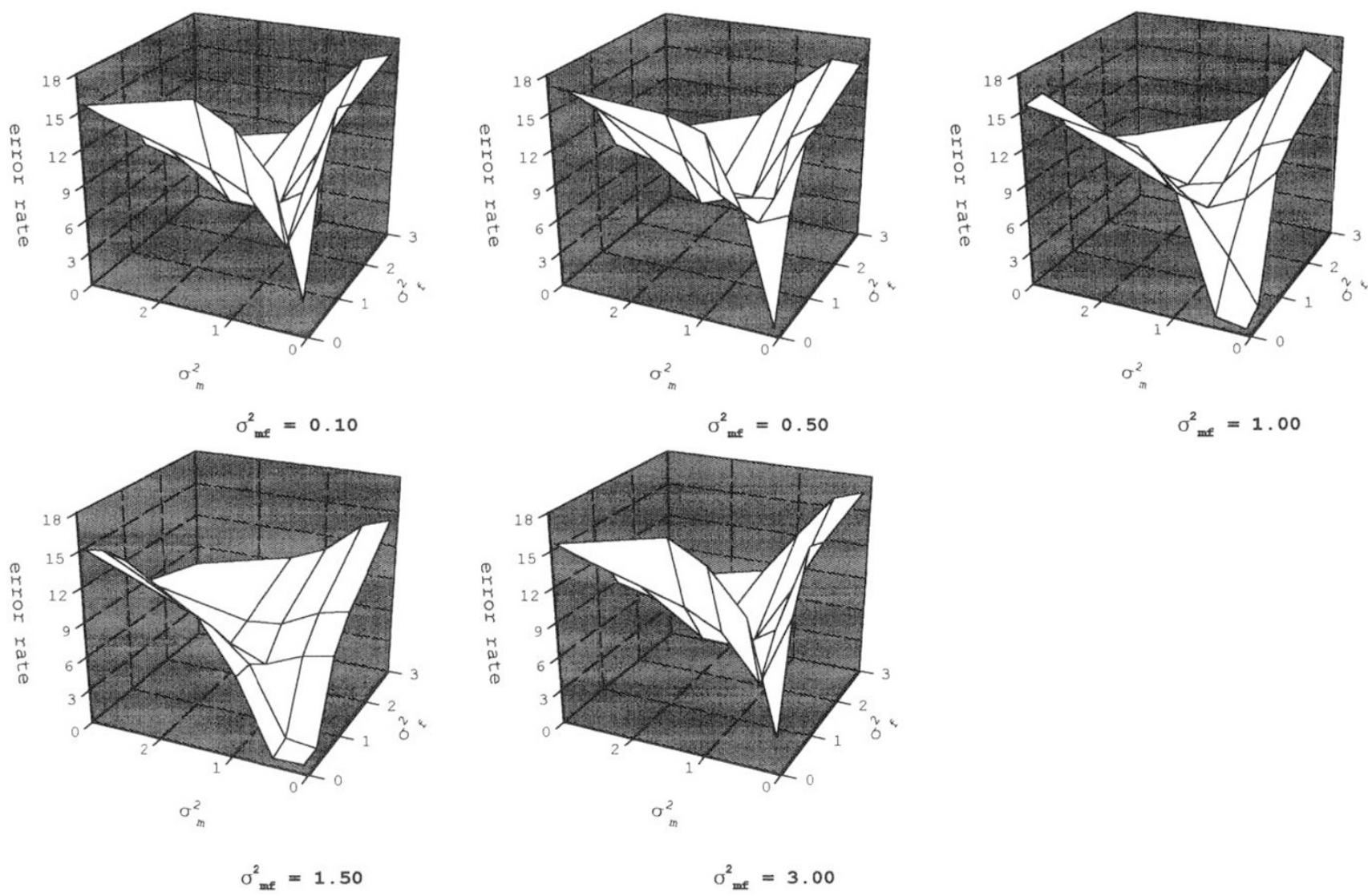

Fig. 4 Relationship between the variance components for females $\left(\sigma_{\mathrm{f}}^{2}\right)$, males $\left(\sigma_{\mathrm{m}}^{2}\right)$ and interactions $\left(\sigma_{\mathrm{mf}}^{2}\right)$ in terms of the error rate (per cent) of heritability estimates $\left(\hat{h}^{2}\right)$ falling outside confidence limits based on the $F$-distribution.

these estimates will be more reliable in setting confidence limits than those based on the formulae of Hallauer \& Miranda (1988), which were derived using Satterthwaite's (1946) estimate of the sampling variance of a variance component.

The SEs produced by the formulae in Appendix 2, adapted from those presented by Hallauer \& Miranda (1988), are unsuitable for setting confidence limits to $\hat{V}_{\mathrm{A}}, \hat{V}_{\mathrm{D}}$ and $\hat{h}^{2}$. The error rates of $\hat{V}_{\mathrm{A}}$ and $\hat{V}_{\mathrm{D}}$ (Fig. 2, Table 3), showed that their SEs were underestimated by formulae (A2.1) and (A2.2). The SEs generated by formula (A2.3) were inflated to such an extent (Tables 4 and 5) that the resulting confidence intervals, assuming a Normal distribution, were far too wide. This resulted in none of the $\hat{h}^{2}$ falling outside the confidence limits.

Confidence limits to $\hat{h}^{2}$ set using the method of Knapp (1986), which is based on the $F$-distribution, were marginally conservative. These conservative confidence limits rendered unacceptably high error rates (Fig. 4).
Thus the 'delta' technique (Taylor approximation) has been shown to provide a reliable means of indicating the accuracy of genetic variance components and $h^{2}$ estimates obtained from REML analysis of North Carolina design II experiments. Certain statistical software packages, e.g. GENSTAT (1993), provide procedures that enable direct calculation of functions and SEs of functions obtained from a REML analysis. Because $\hat{V}_{\mathrm{A}}$ and $\hat{V}_{\mathrm{D}}$ are merely linear functions and $\hat{h}^{2}$ is a ratio of two linear functions of the variance components, estimates of the appropriate SEs can be easily obtained by the appropriate statistical software procedure.

\section{Acknowledgements}

Professor G. P. Y. Clarke is sincerely thanked for his valuable comments and suggestions on the first draft of this article. Grateful acknowledgement is made to Professor S. J. Knapp for his helpful criticisms and suggestions in reviewing this article. 


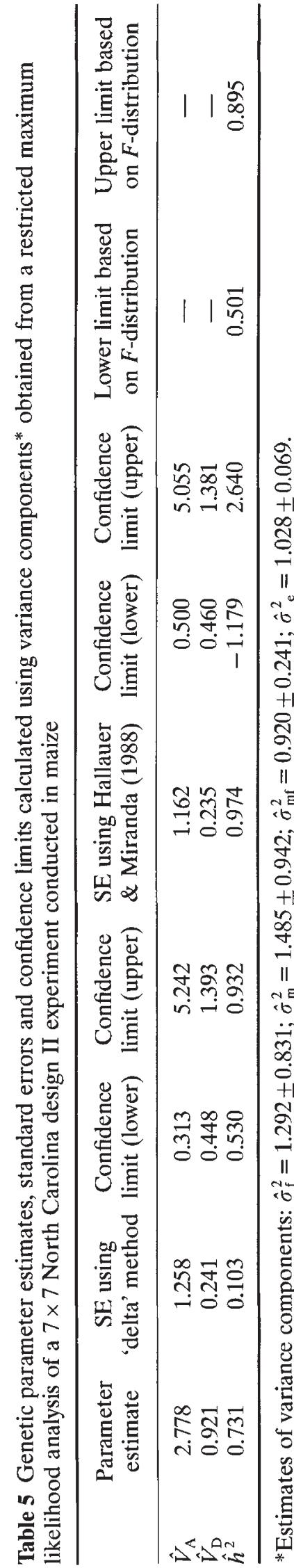

\section{References}

COMSTOCK, R. E. AND ROBINSON, H. F. 1948. The components of genetic variance in populations of biparental progenies and their use in estimating the average degree of dominance. Biometrics, 4, 254-266.

GENSTAT. 1993, Version 5, release 3.1. Lawes Agricultural Trust. Rothamsted Experimental Station, Rothamsted, U.K.

GORDON, 1. L., BYTH, D. E. AND BALAAM, L. N. 1972. Variance of heritability ratios estimated from phenotypic variance components. Biometrics, 28, 401-415.

HALlaUeR, A. R. AND MIRANDA, J. B. 1988. Quantitative Genetics in Maize Breeding. Iowa State University Press, Ames, IA.

HANson, W. D. 1963. Heritability. In: Hanson, W. D. and Robinson, H. F. (eds) Statistical Genetics and Plant Breeding, pp. 125-140. NAS-NRC Publ. 982.

KNAPP, s. J. 1986. Confidence intervals for heritability for two-factor mating design single environment linear models. Theor. Appl. Genet., 72, 587-591.

KNAPP, s. J., STROUP, w. w. AND ROSs, w. M. 1985. Exact confidence intervals for heritability on a progeny mean basis. Crop Sci., 25, 192-194.

MAGNUSSEN, S. 1992. A distribution model for heritability. Genome, 35, 931-938.

NYQUIST, w. E. 1991. Estimation of heritability and prediction of selection response in plant populations. Crit. Rev. Pl. Sci., 10, 235-322.

PATTERSON, H. D. AND THOMPSON, R. 1971. Recovery of inter-block information when block sizes are unequal. Biometrika, 58, 545-554.

ROBINSON, D. L. 1987. Estimation and use of variance components. Statistician, 36, 3-14.

SATTERTHWAITE, F. E. 1946. An approximate distribution of estimates of variance components. Biometrics, 2, $110-114$.

SNEDECOR, G. W. AND COCHRAN, w. G. 1989. Statistical Methods, 8th edn. Iowa State University Press, Ames, IA.

stUART, A. AND ORD, J. K. 1987. Kendall's Advanced Theory of Statistics, vol 1, 5th edn. Oxford University Press, New York.

THOMPSON, R. AND WELHAM, s. J. 1993. REML estimation of variance components and analysis of unbalanced designs. In: GENSTAT 5 committee (eds) GENSTAT 5 Release 3 Reference Manual, pp. 539-584. Clarendon Press, Oxford. 


\section{Appendix 1}

Formulae for the standard errors of $\hat{\mathrm{V}}_{A} \hat{\mathrm{V}}_{D}$ and $\hat{\mathrm{h}}^{2}$, based on the 'delta' technique

The 'delta' technique (Taylor expansion, Stuart and Ord, 1987) may be explained for the function $f$ that has two variables $x$ and $y$, as:

$\operatorname{var} f(x, y) \simeq\left(\frac{\partial f}{\partial x}\right)^{2} \operatorname{var}(x)+\left(\frac{\partial f}{\partial y}\right)^{2} \operatorname{var}(y)+2\left(\frac{\partial f}{\partial x}\right)\left(\frac{\partial f}{\partial y}\right) \operatorname{cov}(x, y)$,

where $\partial f / \partial x$ and $\partial f / \partial y$ are partial derivatives of the function $f(x, y)$ in terms of $x$ and $y$, respectively.

The formulae for the $\operatorname{SE}\left(\hat{V}_{\mathrm{A}}\right)$ and $\operatorname{SE}\left(\hat{V}_{\mathrm{D}}\right)$ are obtained using the 'delta' technique as follows. Using eqn (4), $f\left(\hat{\sigma}_{\mathrm{f}}^{2}, \hat{\sigma}_{\mathrm{m}}^{2}\right)=\hat{\sigma}_{\mathrm{f}}^{2}+\hat{\sigma}_{\mathrm{m}}^{2}$, hence $\partial f / \partial\left(\hat{\sigma}_{\mathrm{f}}^{2}\right)=1=\partial f / \partial\left(\hat{\sigma}_{\mathrm{m}}^{2}\right)$. Thus,

$\operatorname{SE}\left(\hat{V}_{\mathrm{A}}\right)=\sqrt{\operatorname{var}\left(\hat{\sigma}_{\mathrm{f}}^{2}\right)+\operatorname{var}\left(\hat{\sigma}_{\mathrm{m}}^{2}\right)+2 \operatorname{cov}\left(\hat{\sigma}_{\mathrm{f}}^{2}, \hat{\sigma}_{\mathrm{m}}^{2}\right)}$.

Using eqn (5), $f\left(\hat{\sigma}_{\mathrm{mf}}^{2}\right)=\hat{\sigma}_{\mathrm{mf}}^{2}$, and $\partial f / \partial\left(\hat{\sigma}_{\mathrm{mf}}^{2}\right)=1$. Thus,

$\operatorname{SE}\left(\hat{V}_{\mathrm{D}}\right)=\sqrt{\operatorname{var}\left(\hat{\sigma}_{\mathrm{mf}}^{2}\right)}$.

Note that the variance-covariance matrix of the variance components is obtained from the REML analysis.

The formula for the $\operatorname{SE}\left(\hat{h}^{2}\right)$ is derived as follows.

Using $\hat{h}^{2}=\hat{V}_{\mathrm{A}} / \hat{V}_{\mathrm{P}}=f\left(\hat{V}_{\mathrm{A}}, \hat{V}_{\mathrm{P}}\right)$, hence $\partial f / \partial\left(\hat{V}_{\mathrm{A}}\right)=1 / \hat{V}_{\mathrm{P}}$ and $\partial f / \partial\left(\hat{V}_{\mathrm{P}}\right)=-\hat{V}_{\mathrm{A}} / \hat{V}_{\mathrm{P}}^{2}$.

$\operatorname{SE}\left(\hat{h}^{2}\right)=\operatorname{SE}\left(\frac{\hat{V}_{\mathrm{A}}}{\hat{V}_{\mathrm{P}}}\right)$

$$
\begin{aligned}
& =\sqrt{\frac{\operatorname{var}\left(\hat{V}_{\mathrm{A}}\right)}{\hat{V}_{\mathrm{P}}^{2}}-2\left(\frac{\hat{V}_{\mathrm{A}}}{\hat{V}_{\mathrm{P}}^{3}}\right) \operatorname{cov}\left(\hat{V}_{\mathrm{A}}, \hat{V}_{\mathrm{P}}\right)+\left(\frac{\hat{V}_{\mathrm{A}}^{2}}{\hat{V}_{\mathrm{P}}^{4}}\right)\left(\operatorname{var}\left(\hat{V}_{\mathrm{P}}\right)\right)} \\
& =\left(\frac{1}{\hat{V}_{\mathrm{P}}}\right) \sqrt{\operatorname{var}\left(\hat{V}_{\mathrm{A}}\right)-2\left(\frac{\hat{V}_{\mathrm{A}}}{\hat{V}_{\mathrm{P}}}\right) \operatorname{cov}\left(\hat{V}_{\mathrm{A}}, \hat{V}_{\mathrm{P}}\right)+\left(\frac{\hat{V}_{\mathrm{A}}}{\hat{V}_{\mathrm{P}}}\right)^{2}\left(\operatorname{var}\left(\hat{V}_{\mathrm{P}}\right)\right)}
\end{aligned}
$$

The $\operatorname{var}\left(\hat{V}_{\mathrm{P}}\right)$ is obtained by using the 'delta' technique as follows. Using $\hat{V}_{\mathrm{P}}=\hat{\sigma}_{\mathrm{f}}^{2}+\hat{\sigma}_{\mathrm{m}}^{2}+\hat{\sigma}_{\mathrm{mf}}^{2}+\hat{\sigma}_{\mathrm{e}}^{2} / r$ $=f\left(\hat{\sigma}_{\mathrm{f}}^{2}, \hat{\sigma}_{\mathrm{m}}^{2}, \hat{\sigma}_{\mathrm{mf}}^{2}, \hat{\sigma}_{\mathrm{e}}^{2}\right)$, hence $\partial f / \partial\left(\hat{\sigma}_{\mathrm{f}}^{2}\right)=\partial f / \partial\left(\hat{\sigma}_{\mathrm{m}}^{2}\right)=\partial f / \partial\left(\hat{\sigma}_{\mathrm{mf}}^{2}\right)=1$ and $\partial f / \partial\left(\hat{\sigma}_{\mathrm{e}}^{2}\right)=1 / r$. Thus,

$$
\begin{aligned}
\operatorname{var}\left(\hat{V}_{\mathrm{P}}\right)= & \operatorname{var}\left(\hat{\sigma}_{\mathrm{f}}^{2}\right)+\operatorname{var}\left(\hat{\sigma}_{\mathrm{m}}^{2}\right)+\operatorname{var}\left(\hat{\sigma}_{\mathrm{mf}}^{2}\right)+\left(\frac{1}{r}\right)^{2} \operatorname{var}\left(\hat{\sigma}_{\mathrm{e}}^{2}\right) \\
& +2 \operatorname{cov}\left(\hat{\sigma}_{\mathrm{f}}^{2}, \hat{\sigma}_{\mathrm{m}}^{2}\right)+2 \operatorname{cov}\left(\hat{\sigma}_{\mathrm{f}}^{2}, \hat{\sigma}_{\mathrm{mf}}^{2}\right)+\left(\frac{2}{r}\right) \operatorname{cov}\left(\hat{\sigma}_{\mathrm{f}}^{2}, \hat{\sigma}_{\mathrm{e}}^{2}\right) \\
& +2 \operatorname{cov}\left(\hat{\sigma}_{\mathrm{m}}^{2}, \hat{\sigma}_{\mathrm{mf}}^{2}\right)+\left(\frac{2}{r}\right)+\left(\frac{2}{r}\right) \operatorname{cov}\left(\hat{\sigma}_{\mathrm{m}}^{2}, \hat{\sigma}_{\mathrm{e}}^{2}\right) \operatorname{cov}\left(\hat{\sigma}_{\mathrm{mf}}^{2}, \hat{\sigma}_{\mathrm{e}}^{2}\right) .
\end{aligned}
$$

For two linear functions, $f(x, y)$ and $g(x, y)$, the Taylor expansion gives:

$\operatorname{cov}(f(x, y), g(x, y)) \simeq\left(\frac{\partial f}{\partial x}\right)\left(\frac{\partial g}{\partial x}\right) \operatorname{var}(x)+\left(\frac{\partial f}{\partial y}\right)\left(\frac{\partial g}{\partial y}\right) \operatorname{var}(y)$ 


$$
+\left(\frac{\partial f}{\partial x}\right)\left(\frac{\partial g}{\partial y}\right) \operatorname{cov}(x, y)+\left(\frac{\partial f}{\partial y}\right)\left(\frac{\partial g}{\partial x}\right) \operatorname{cov}(x, y)
$$

Using equation (4), $f\left(\hat{\sigma}_{\mathrm{f}}^{2}, \hat{\sigma}_{\mathrm{m}}^{2}\right)=\hat{\sigma}_{\mathrm{f}}^{2}+\hat{\sigma}_{\mathrm{m}}^{2}$, hence $\partial f / \partial\left(\hat{\sigma}_{\mathrm{f}}^{2}\right)=1=\partial f / \partial\left(\hat{\sigma}_{\mathrm{m}}^{2}\right)$ and $\partial f / \partial\left(\hat{\sigma}_{\mathrm{mf}}^{2}\right)=\partial f / \partial\left(\hat{\sigma}_{\mathrm{e}}^{2}\right)=0$; and $g\left(\hat{\sigma}_{\mathrm{f}}^{2}, \hat{\sigma}_{\mathrm{m}}^{2}, \hat{\sigma}_{\mathrm{mf}}^{2}, \hat{\sigma}_{\mathrm{e}}^{2}\right)=\hat{\sigma}_{\mathrm{f}}^{2}+\hat{\sigma}_{\mathrm{m}}^{2}+\hat{\sigma}_{\mathrm{mf}}^{2}+\hat{\sigma}_{\mathrm{e}}^{2} / r$, hence $\partial g / \partial\left(\hat{\sigma}_{\mathrm{f}}^{2}\right)=\partial g / \partial\left(\hat{\sigma}_{\mathrm{m}}^{2}\right)=\partial g / \partial\left(\hat{\sigma}_{\mathrm{mf}}^{2}\right)=1$ and $\partial g / \partial\left(\hat{\sigma}_{\mathrm{e}}^{2}\right)=1 / r$

Thus, $\operatorname{cov}\left(\hat{V}_{\mathrm{A}}, \hat{V}_{\mathrm{P}}\right)=\operatorname{var}\left(\hat{\sigma}_{\mathrm{f}}^{2}\right)+\operatorname{var}\left(\hat{\sigma}_{\mathrm{m}}^{2}\right)+2 \operatorname{cov}\left(\hat{\sigma}_{\mathrm{f}}^{2}, \hat{\sigma}_{\mathrm{m}}^{2}\right)+\operatorname{cov}\left(\hat{\sigma}_{\mathrm{f}}^{2}, \hat{\sigma}_{\mathrm{mf}}^{2}\right)+\left(\frac{1}{r}\right) \operatorname{cov}\left(\hat{\sigma}_{\mathrm{f}}^{2}, \hat{\sigma}_{\mathrm{e}}^{2}\right)$

$$
+\operatorname{cov}\left(\hat{\sigma}_{\mathrm{m}}^{2}, \hat{\sigma}_{\mathrm{mf}}^{2}\right)+\left(\frac{1}{r}\right) \operatorname{cov}\left(\hat{\sigma}_{\mathrm{m}}^{2}, \hat{\sigma}_{\mathrm{e}}^{2}\right)
$$

\section{Appendix 2}

Formulae for the standard errors of $\hat{\mathrm{V}}_{A}, \hat{\mathrm{V}}_{D}$ and $\hat{\mathrm{h}}^{2}$, based on equations given by Hallauer \& Miranda (1988) Using Satterthwaite's (1946) formula (formula (7)) for estimating sampling variances of variance components:

$\mathrm{SE}\left(\hat{V}_{\mathrm{A}}\right)=\sqrt{\frac{8}{(r a)^{2}}\left[\frac{M_{\mathrm{m}+\mathrm{f}}^{2}}{(a+b)}+\frac{M_{\mathrm{mf}}^{2}}{[(a-1)(b-1)+2]}\right]}$,

$\mathrm{SE}\left(\hat{V}_{\mathrm{D}}\right)=\sqrt{\frac{2}{(r)^{2}}\left[\frac{M_{\mathrm{mf}}^{2}}{[(a-1)(b-1)+2]}+\frac{M_{\mathrm{e}}^{2}}{[(a b(r-1)+2]}\right]}$,

where $a$ and $b$ are the numbers of female and male parents, respectively, and $r$ is the number of observations per cross; $M_{\mathrm{m}+\mathrm{f}}, M_{\mathrm{mf}}$ and $M_{\mathrm{e}}$ are the Mean Squares for the males and females, males $\times$ females and withinfamily sources of variation, respectively (Table 1 ).

$\operatorname{SE}\left(\hat{h}^{2}\right)=\frac{2 \operatorname{SE}\left(\hat{\sigma}_{\mathrm{m}+\mathrm{f}}^{2}\right)}{\frac{\hat{\sigma}_{\mathrm{e}}^{2}}{r}+\hat{\sigma}_{\mathrm{mf}}^{2}+2 \hat{\sigma}_{\mathrm{m}+\mathrm{f}}^{2}}$.

An estimate of the $\operatorname{SE}\left(\hat{\sigma}_{\mathrm{m}+\mathrm{f}}^{2}\right)$ is obtained from the relationship:

$\hat{\sigma}_{\mathrm{m}+\mathrm{f}}^{2}=\left(M_{\mathrm{m}+\mathrm{f}}-M_{\mathrm{mf}}\right) /[r(a+b) / 2]$,

and using formula (7), as:

$\mathrm{SE}\left(\hat{\sigma}_{\mathrm{m}+\mathrm{f}}^{2}\right)=\sqrt{\frac{2}{(r a)^{2}}\left[\frac{M_{\mathrm{m}+\mathrm{f}}^{2}}{(a+b)}+\frac{M_{\mathrm{mf}}^{2}}{[(a-1)(b-1)+2]}\right]}$. 